\title{
SARS-CoV-2 takes its Toll
}

\author{
SARS-CoV-2 infection activates TLR2 signaling, which results in the robust expression of proinflammatory \\ cytokines that may contribute to disease in severe COVID-19. Inhibition of this signaling pathway represents a \\ potential target for COVID-19 therapeutics.
}

\section{Alan Sariol and Stanley Perlman}

$\mathrm{S}$ evere disease in COVID-19 is associated with an inappropriately regulated cytokine response that results in immune-mediated pathology of the lungs and other tissues. While myeloid cells, including monocytes and macrophages, are known to contribute to this immunopathology ${ }^{1}$, the upstream signaling pathways that activate this hyperactive cytokine response have not been fully described. In this issue of Nature Immunology, Zheng and colleagues ${ }^{2}$ identify Toll-like receptor (TLR)2 as a sensor of SARS-CoV- 2 and other $\beta$-coronaviruses that drives inflammatory cytokine production, potentially contributing to the dysregulated immune response observed in patients with severe COVID-19.

Seeking a mechanistic explanation for the robust expression of proinflammatory cytokines in COVID-19, the authors investigated TLR activation, as the TLR adaptor MYD88 has been identified as a critical factor in the production of a number of inflammatory cytokines in $\beta$-coronavirus infections $s^{3,4}$. In a cohort of patients with COVID-19 stratified by disease severity, they found that mRNA expression levels of Myd88, along with those of several TLRs that signal through it, were directly correlated with disease severity, suggesting that signaling through this adaptor may be involved in pathogenesis.

To investigate this further, Zheng et al. used both SARS-CoV-2 and mouse hepatitis virus (MHV), the prototypical laboratory coronavirus and a fellow member of the Betacoronavirus genus that causes a variety of diseases in susceptible rodents ${ }^{5}$. Infection of $\mathrm{MyD} 88^{-/-}$mouse bone-marrow-derived macrophages with MHV resulted in a substantial reduction in TNF expression relative to wild-type (WT) controls, further highlighting the role of TLRs in sensing $\beta$-coronavirus infection. In order to identify which TLRs were required for this signaling, macrophages deficient in TLRs 2, 4, 7 or 9 were infected with MHV. The expression of Tnf, Il6 and other inflammatory cytokine genes was abrogated in cells deficient in
TLR2, while other TLR deficiencies had minimal effect on the expression of these genes. For SARS-CoV-2, they then found that treatment of infected human peripheral blood mononuclear cells (PBMCs) with an inhibitor of TLR2, but not TLR4, resulted in significantly reduced cytokine and chemokine production, further confirming that TLR2 plays a critical role in sensing these viruses upstream of MYD88 (Fig. 1a).

Zheng and colleagues next sought to identify the component of these viruses that activates TLR2. In a series of experiments using heat-inactivated virus to block transcription and entry, they determined that viral replication is not required for TLR2-mediated activation of inflammatory signaling, suggesting that the component involved in TLR2 activation is a structural protein found in the virion, rather than a non-structural protein expressed only within infected cells. With this in mind, they then focused on 3 of the 4 major structural proteins found in all coronaviruses, the spike (S), envelope (E) and membrane (M) (Fig. 1b) proteins, to identify the component of the virion that activates TLR2. Using purified recombinant proteins, they identified SARS-CoV-2 E protein as a TLR2-dependent activator of inflammatory signaling pathways in mouse bone-marrow-derived macrophages and human PBMCs, whereas no activation was observed in response to the $\mathrm{S}$ or $\mathrm{M}$ proteins. Using coimmunoprecipitation, they also observed that recombinant $\mathrm{E}$ protein interacted directly with TLR2, further suggesting that the E protein can activate TLR2.

Taking these observations to in vivo infection models, the authors found that intratracheal administration of SARS-CoV-2 E protein to mice could induce cell death and inflammatory cytokine production in the lungs, similar to the effects induced by a known TLR2 agonist, whereas these effects were abrogated in the lungs of Tlr $2^{-/-}$mice. Finally, the authors sought to determine the impact of this TLR2-mediated signaling on disease pathogenesis in transgenic

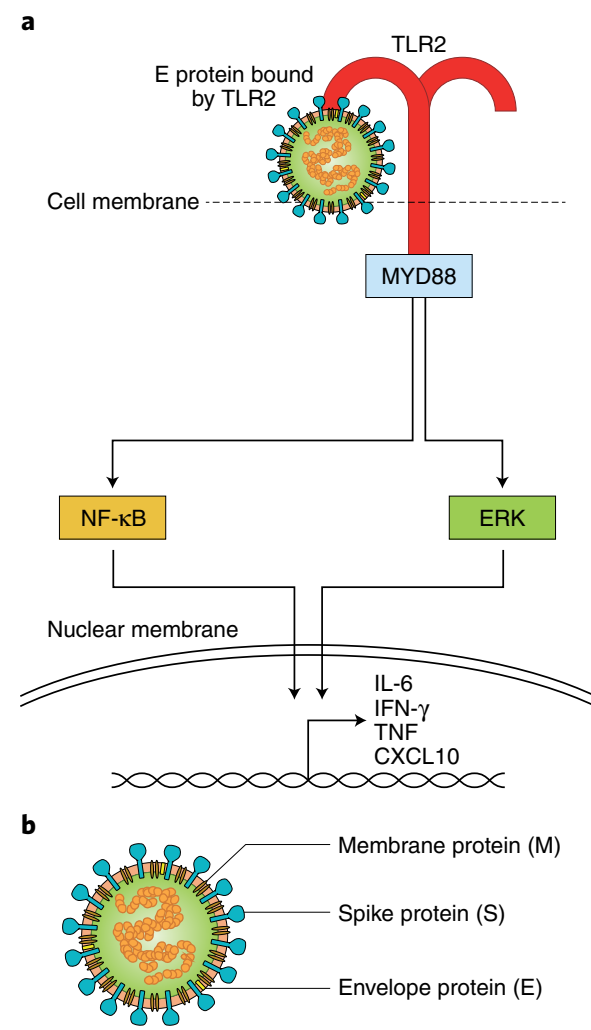

Fig. 1 | SARS-CoV-2 signals through TLR2 to activate proinflammatory cytokine expression. a, Simplified TLR2 signaling pathway. In brief, the envelope proteins of SARS-CoV-2 and other $\beta$-coronaviruses signal through TLR2 to activate the adaptor MYD88, which subsequently signals through NF- $\mathrm{kB}$ and ERK to promote the expression of inflammatory cytokines. $\mathbf{b}$, Schematic representation of a SARS-CoV-2 virion, with major structural proteins on the virion surface labeled.

mice expressing the human SARS-CoV-2 entry receptor ${ }^{6,7}$. Administration of a TLR2 inhibitor on day 0 and day 3 post-infection resulted in a modest enhancement of survival and a significant reduction in the production of inflammatory cytokines such as interleukin-6, tumor necrosis factor and interferon- $\gamma$, suggesting that TLR2-mediated 
inflammation plays a pathogenic role in SARS-CoV-2 infection.

Together, these studies describe a heretofore unidentified sensor of coronaviruses and provide insight into the mechanisms behind the robust inflammatory cytokine production that contributes to pathogenesis in COVID-19 and other $\beta$-coronavirus-mediated diseases, such as SARS and MERS. While previous studies of MHV and SARS-CoV have identified cytosolic sensors of coronaviral RNA, such as Mda5 (ref. ${ }^{8}$ ), as well as TLR7 activation in plasmacytoid dendritic cells that results in a robust type I interferon response ${ }^{9}$, TLR2 activation is a new mechanism of coronavirus sensing. Unlike TLR7 activation in plasmacytoid dendritic cells, which protects against infection, TLR2 activation was observed in human PBMCs, which primarily consist of monocytes, and mouse macrophages-cell types that are thought to contribute to inflammatory disease pathogenesis in COVID-19. These findings may thus represent a mechanism by which these cells contribute to the exuberant cytokine secretion that is associated with severe COVID-19.

By contrast, in a preprint study ${ }^{10}$, another group has identified the $S$ protein, and not the E protein, as a driver of TLR2 activation and subsequent cytokine production in SARS-CoV-2 infection of human and mouse macrophages. This further corroborates the finding that TLR2 signaling drives inflammation following SARS-CoV-2 infection. In addition, the $\mathrm{E}$ protein is embedded in the membrane of the virion, with little exposure to the extracellular milieu, while the $\mathrm{S}$ protein is more obviously exposed. While the topology of the $\mathrm{E}$ protein in the SARS-CoV-2 virion has not yet been fully elucidated, data from other $\beta$-coronaviruses, such as MHV, suggest that there may only be a short, 7-amino-acid hydrophilic N-terminal domain exposed outside of the virion, with the remainder of the 75-amino-acid protein comprising a transmembrane domain and a hydrophilic $\mathrm{C}$ terminus exposed to the virion interior ${ }^{11}$. Supporting this, evidence suggests that the $\mathrm{C}$ terminus of the SARS-CoV-2 E protein is exposed cytoplasmically in eukaryotic cell membranes ${ }^{12}$. Together, these results indicate that identification of the precise viral protein(s) involved in TLR2 activation and the mechanisms by which the E protein functions in this capacity require further investigation to understand these seemingly disparate results.

This study highlights TLR2 as a potential target for therapeutics for both COVID-19 and other coronavirus-induced diseases, as therapeutics targeting the immune response are critical for mitigating severe disease. On the other hand, in the setting of the very mild SARS-CoV-2 infection observed in ferrets, prophylactic treatment with a TLR2 agonist reduced viral titers in the nasopharyngeal and oral cavities without inducing significant clinical signs of inflammation, suggesting that TLR2 engagement can also play a protective role in the right setting ${ }^{13}$. It is possible that, similar to results observed with type I interferon ${ }^{14}$, the timing of TLR2 activation is a critical factor for the development of pathogenic or protective responses, with early TLR2 activation potentially conferring protection and delayed signaling resulting in a dysregulated, pathogenic response. As is true for many therapies suggested for use in COVID-19, identifying patients who are likely to progress to more severe disease would inform the use of agents targeting TLR2 signaling.

\section{Alan Sariol ${ }^{1}$ and Stanley Perlman (D) $2 \bowtie$ ${ }^{1}$ Department of Microbiology and Immunology, University of Iowa, Iowa City, IA, USA. ${ }^{2}$ Interdisciplinary Program in Immunology, University of Iowa, Iowa City, IA, USA.

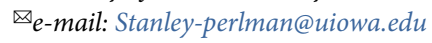

Published online: 8 June 2021

https://doi.org/10.1038/s41590-021-00962-w

References

1. Merad, M. \& Martin, J. C. Nat. Rev. Immunol. 20, 355-362 (2020).

2. Zheng, M. et al. Nat. Immunol. https://doi.org/10.1038/s41590-

021-00937-x (2021).

3. Zhou, H., Zhao, J. \& Perlman, S. Mbio 1, e00219-10 (2010).

4. Sheahan, T. et al. PLoS Pathog. 4, e1000240 (2008).

5. Barthold, S. W. \& Smith, A. L. Arch. Virol. 81, 103-112 (1984).

6. Winkler, E. S. et al. Nat. Immunol. 21, 1327-1335 (2020).

7. Zheng, J. et al. Nature 589, 603-607 (2021).

8. Roth-Cross, J. K., Bender, S. J. \& Weiss, S. R. J. Virol. 82, 9829-9838 (2008).

9. Cervantes-Barragan, L. et al. Blood 109, 1131-1137 (2007).

10. Khan, S. et al. Preprint at bioRxiv https://doi. org/10.1101/2021.03.16.435700 (2021).

11. Maeda, J., Repass, J. F., Maeda, A. \& Makino, S. Virology 281, 163-169 (2001).

12. Duart, G. et al. Open Biol. 10, 200209 (2020).

13. Proud, P. C. et al. EBioMedicine 63, 103153 (2021).

14. Channappanavar, R. et al. J. Clin. Invest. 129, 3625-3639 (2019).

The authors declare no competing interests.

\section{"Mind the GAP": RGS1 hinders antitumor lymphocytes}

Effective anticancer adoptive cellular therapy (ACT) requires sufficient infiltration of injected cytotoxic lymphocytes, but pathophysiology frustrates this. Regulator of $G$ protein signaling 1 limits $T$ cell trafficking to breast tumors and may be targeted to improve ACT.

\section{Frédéric Fercoq and Leo M. Carlin}

reast cancers are often considered immunologically cold, with low lymphocyte infiltration leading to poor responses to immune checkpoint inhibitors (ICI), such as anti-PD-1 or anti-PD-L1 (ref. ${ }^{1}$ ). Adoptive cellular therapy (ACT), which involves the infusion of expanded and manipulated T cells, aims to provide an abundant population of tumor-reactive $\mathrm{T}$ cells to increase antitumor immune responses, but this can be frustrated by the way immunity is regulated. In the current issue of Nature Immunology, Huang et al. $^{2}$ describe a new mechanism by which antitumor $\mathrm{T}$ cell migration is inhibited. They 